\title{
Establishment of Thermal Comfort Evaluation Model Based on Individual Difference
}

\author{
Yalong Yang ${ }^{1,2,3}$, Yufu Liu ${ }^{1,3}$, Rui Zhang ${ }^{1,3}$, Xulai Zhu ${ }^{1,3}$ and Mingyue Wang ${ }^{1,2,3, *}$ \\ ${ }^{1}$ Anhui Province Key Laboratory of Intelligent Building and Building Energy Saving, Anhui Jianzhu University, 230022, Hefei, China \\ ${ }^{2}$ Key Laboratory of Huizhou Architecture in Anhui Province, Anhui Jianzhu University, 230022, Hefei, China \\ ${ }^{3}$ School of Electronic and Information Engineering, Anhui Jianzhu University, 230601, Hefei, China
}

\begin{abstract}
People-oriented" and "Energy saving" are the two major themes of current social development. In the field of thermal comfort, establishment of thermal comfort model based on physiological parameters plays an important role in meeting the needs of human health and comfort, optimizing the design of building environment and building energy saving. In this paper, three types of physiological signals (skin temperature, skin conductance and heart rate) were collected through comfort physiological experiments. The changes of the three types of physiological signals under environmental temperature were analyzed. Furthermore, subjective questionnaire survey of human thermal comfort under five experimental conditions was performed. In addition, the thermal comfort evaluation model based on individual differences was established by partial least squares regression and ELM-RBF neural network. The established models were compared with the classical PMV model to analyze the superiority of the model. The results show that the thermal comfort evaluation model based on individual differences established by ELM-RBF neural network can better predict the trend of people's thermal comfort and satisfy the individual's demand for thermal comfort. Meanwhile, it can achieve the goal of building energy saving. Therefore, it has high practical and social significance.
\end{abstract}

\section{Introduction}

The indoor environment affecting the comfort of personnel mainly includes heat, sound, light environment and air quality, and the influence of thermal environment is most significant[1]. The study of human body thermal comfort is divided into two categories, one is the experimental method, which explores the influence factors of human body thermal comfort from the aspects of environment, human physiology and psychology. The second is the model method, which puts forward the means and methods to improve the thermal comfort of the human body according to the prediction of the thermal comfort of the human body under different environmental conditions[2]. Air conditioning provides constant temperature and humidity to improve indoor thermal comfort[3]. However, the use of traditional air conditioners can impair people's thermal adaptability[4]. In recent years, researchers have used physiology to study thermal comfort and have predicted and evaluated the body's thermal comfort levels under different environmental conditions by building thermal comfort models[5]. It provides a reliable means for comprehensive study of the relationship between human thermal comfort and influencing factors[6]. Thermal comfort research involves physiology, psychology, ecology and so on. Accurate evaluation of human thermal comfort state is the basis for thermal comfort research[7]. The thermal comfort model is based on the physiological characteristics of the human body, and the variability of different individuals is not the same[8]. Professor Qiansheng Fang from Anhui Jianzhu University established a simulation model of temperature and humidity control system, a feed-forward decoupler with Smith prediction link was designed and the control algorithm of the system was improved and a dynamic thermal comfort control method was presented [9]. Ali Ghahramani proposed a novel human thermal comfort monitoring method for individual differences in thermal comfort requirements [10]. Since some physiological parameters appear linear change severity with the change of environment, and some physiological parameters show the law of nonlinear change with the change of environment. Therefore, the thermal comfort evaluation model based on individual differences is established by using the methods of partial least squares regression and ELM-RBF neural network in this paper, and the model is verified by further experiments. The established model meets the thermal comfort needs of the human and can achieve the goal of building energy saving. 


\section{Methods}

\subsection{Partial Least Squares Regression}

Taking the influence factor of human thermal comfort as an example, there are six research objects written as $\left\{X_{1}, X_{2}, \cdots, X_{6}\right\}$, which represent the ambient temperature, skin temperature, heart rate, skin conductance, body type and gender. The influencing factor of human thermal comfort was considered as the dependent variable $Y$, which corresponds to the independent variable $X$. In order to establish the relationship between the human thermal comfort value and the six influencing factors, the principal components are extracted in $X$ and $Y$ respectively.

Assuming $\left\{t_{1}, t_{2}, \cdots, t_{r}\right\}$ is the main component of $\left\{X_{1}, X_{2}, \cdots, X_{6}\right\}, u_{1}$ is the principal component of $Y$, $r \leq 6$, it needs to meet the following requirements:

(1) $t_{1}$ and $u_{1}$ should be able to carry the variability data to the maximum extent;

(2) The correlation between $t_{1}$ and $u_{1}$ needs to be as high as possible.

As a result, $t_{1}$ and $u_{1}$ should be able to represent the original data $X$ and $Y$ as much as possible, and the principal components $u_{1}$ of dependent variable can be explained greatly by the principal component $t_{1}$ of independent variable.

The specific algorithm implementation is as follows:

(1) After the source data having been normalized, matrix $X_{0}$ can be obtained.

$$
X_{0}=\left[\begin{array}{cccc}
x_{11} & x_{12} & \cdots & x_{16} \\
x_{21} & x_{22} & \cdots & x_{26} \\
\vdots & \vdots & \ddots & \vdots \\
x_{n 1} & x_{n 2} & \cdots & x_{n 6}
\end{array}\right]
$$

Furthermore, the observation matrix $Y_{0}$ can be obtained.

$$
Y_{0}=\left[\begin{array}{cccc}
y_{11} & y_{12} & \cdots & y_{16} \\
y_{21} & y_{22} & \cdots & y_{26} \\
\vdots & \vdots & \ddots & \vdots \\
y_{n 1} & y_{n 2} & \cdots & y_{n 6}
\end{array}\right]
$$

(2) Finding the first principal component of the $X$ group variable $t_{1}, w_{1}$ is the coefficient vector of the first principal component, and $w_{1}$ is the unit vector.

$$
t_{1}=X_{0} w_{1}
$$

Finding the first principal component of the $Y$ group variable $t_{1}$, unit vector $c_{1}$ is the coefficient vector of the first principal component, $\operatorname{Var}\left(t_{1}\right)=\max$,
$\operatorname{Var}\left(u_{1}\right)=\max$ and $\rho\left(t_{1}, u_{1}\right)=\max$, formula (4) and formula (5) can be obtained.

$$
\begin{gathered}
u_{1}=Y_{0} c_{1} \\
\left\{\begin{array}{l}
\max _{w_{1}, c_{1}}\left(X_{0} w_{1}, Y_{0} c_{1}\right) \\
w_{1}^{\prime} w_{1}=1 \\
c_{1}^{\prime} c_{1}=1
\end{array}\right.
\end{gathered}
$$

(3) Establishment of regression equation.

The two regression equations are as follows.

$$
\begin{gathered}
X_{0}=t_{1} \alpha_{1}^{\prime}+E_{1} \\
Y_{0}=t_{1} \beta_{1}^{\prime}+F_{1}
\end{gathered}
$$

$\alpha_{1}$ - The amount of load for the model effect.

All the information of the first pair of principal components can't be extracted, and $\alpha_{2}, \alpha_{3}, \ldots, \alpha_{r}$ can't be extracted, either.

(4) Assuming the rank of the $n \times 6$ matrix is $r=\min (n, 6)$, there are $t_{1}, t_{2}, \ldots, t_{r}$ satisfy equation (8).

$$
\begin{gathered}
\left\{\begin{array}{l}
X_{0}=t_{1} \alpha_{1}^{\prime}+t_{2} \alpha_{2}^{\prime}+\cdots+E_{r} \\
Y_{0}=t_{1} \beta_{1}^{\prime}+t_{2} \beta_{2}^{\prime}+\cdots+F_{r}
\end{array}\right. \\
t_{k}=w_{k 1} X_{1}+w_{k 2} X_{2}+\cdots+w_{k p} X_{p}, k=1,2, \ldots r
\end{gathered}
$$

Substituting (9) into (8), and combining the same items.

$$
\hat{Y}_{j}=a_{j 1} X_{1}+a_{j 2} X_{2}+\cdots a_{j 6} X_{6}
$$

The non-standardized partial least squares equation is as follows:

$$
\hat{Y}_{j}=a_{j 0}+a_{j 1} X_{1}+a_{j 2} X_{2}+\cdots a_{p} X_{p}
$$

\subsection{ELM-RBF Neural Network}

The RBF neuron was replaced with ELM-RBF neural network, which is a single hidden layer feed-forward neural network. The advantage is that the computing speed is fast and the nonlinear fitting result is satisfactory. The structure of ELM-RBF neural network topology is as shown in Figure 1.

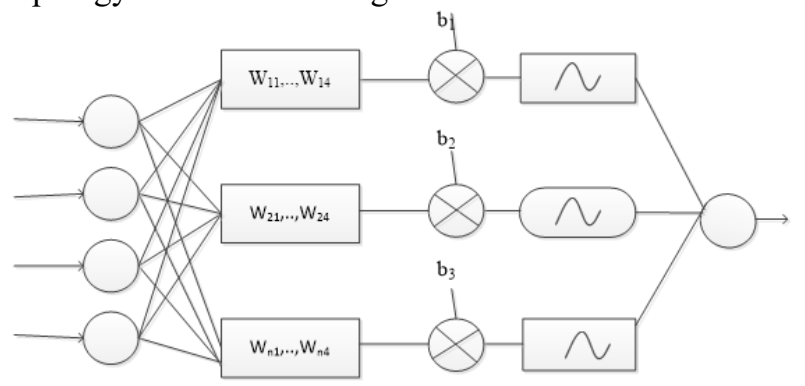

Figure. 1. ELM-RBF neural network topology.

The output is as shown in equation (12).

$$
f\left(x_{i}\right)=\sum_{j=1}^{N} \beta_{j} \varphi_{j}\left(x_{i}\right)
$$


where,

$x_{i}$ - The ith input vector;

$f\left(x_{i}\right)$ - The output of the topology map through the network;

$\beta_{j}$ - The weight of the jth kernel function connected to the output;

$N$ - The number of kernel functions;

$\varphi_{j}\left(x_{i}\right)$ - The output of the jth kernel function.

In this paper, the kernel function is a Gaussian function.

$$
\varphi_{j}\left(x_{i}\right)=\varphi\left(u_{j}, \sigma_{j}, x_{j}\right)=\operatorname{Exp}\left(-\frac{\left\|x_{i}-w_{j}\right\|}{b_{j}^{2}}\right)
$$

In equation (13),

$w_{j}$ - The weight vector of the kth kernel function;

$b_{j}$ - The threshold of the kth kernel function.

Clearly, when $x_{i}=x_{j}$, the maximum value of $\varphi_{j}\left(x_{i}\right)$ can be realized.

For arbitrary sampling points $\left(x_{i}, y_{i}\right)(1 \leq i \leq M)$, assuming a sequence of RBF kernel functions $\varphi_{N}$, its weight vector $w_{j}$ and threshold $b_{j}$ are randomly generated according to any sampling distribution probability, there must be a positive number $Z$, an arbitrarily small $\delta$ and an output weight vector $\beta_{j}$ satisfy equation (14) when $N=Z$.

$$
\left\|f\left(x_{i}\right)-y_{i}\right\|<\delta
$$

There are a positive number $Z$ and $\beta_{j}$ satisfy equation (15).

$$
\begin{gathered}
y_{i}=\sum_{j=1}^{N} \beta_{i} \varphi_{j}\left(x_{i}\right) \\
H \beta=Y
\end{gathered}
$$

where,

$$
H=\left[\begin{array}{cccc}
\varphi_{1}\left(x_{1}\right) & \varphi_{2}\left(x_{1}\right) & \cdots & \varphi_{N}\left(x_{1}\right) \\
\varphi_{1}\left(x_{2}\right) & \varphi_{2}\left(x_{2}\right) & \cdots & \varphi_{N}\left(x_{2}\right) \\
\vdots & \vdots & \ddots & \vdots \\
\varphi_{1}\left(x_{M}\right) & \varphi_{2}\left(x_{M}\right) & \cdots & \varphi_{2}\left(x_{M}\right)
\end{array}\right]
$$

The algorithm steps are as follows:

(1) Generating an initial population and setting the center and width of a hidden layer neuron in the ELMRBF neural network;

(2) Calculating the output weight of the hidden layer;

(3) Calculating the weight of the output layer.

\section{Experiments of Human Thermal Comfort}

In this paper, the differences of human skin temperature, skin conductance and heart rate at different indoor temperatures were studied. The purpose aims at studying the effects of thermal comfort on human physiology and exploring the changes of physiological parameters and temperature. In addition, in order to establish an evaluation model of human thermal comfort based on individual differences, subjective questionnaires for perspiration, thermal environment expectations, indoor thermal environment and air quality satisfaction were collected.

\subsection{Experimental Environment}

The experimental environment is as shown in Table 1.

Table. 1. Experimental conditions.

\begin{tabular}{|c|l|}
\hline Items & \multicolumn{1}{|c|}{ Parameters } \\
\hline Dimensions of laboratory & $\begin{array}{l}\text { Laboratory: } 8.7 \mathrm{~m} \times 6.4 \mathrm{~m} \times 2.6 \mathrm{~m} \quad \text { (length } \times \text { width } \times \text { height }) \text { Working } \\
\text { area: } 8.4 \mathrm{~m} \times 6.1 \mathrm{~m} \times 2.4 \mathrm{~m}(\text { length } \times \text { width } \times \text { height })\end{array}$ \\
\hline Materials of laboratory & $\begin{array}{l}\text { The inner and outer walls: } 240 \mathrm{~mm} \text { in width } \\
\text { Roof: waterproofing and thermal insulation } \\
\text { Windows: double glass and steel frame }\end{array}$ \\
\hline $\begin{array}{c}\text { Parameters of constant } \\
\text { temperature and humidity air } \\
\text { conditioner }\end{array}$ & $\begin{array}{l}\text { Supply air rate: } \mathrm{Gs}=5200 \mathrm{~m} 3 / \mathrm{h} \\
\text { Air changes: } \mathrm{N}=\mathrm{Gs} / \mathrm{LWH}=40 \text { time/h }\end{array}$ \\
\hline
\end{tabular}

\subsection{Design of Questionnaires}

Subjective parameters used to reflect human thermal comfort include perspiration, thermal environmental expectations, indoor thermal environment and air quality satisfaction can't be measured by instruments, therefore, subjective questionnaires are used to obtain thermal comfort values scientifically and reasonably. The design scale of the questionnaires are as shown in Tables 2-4. In Table 2, a four-stage scale was selected as the perspiration gauge. In Table 3 , a three-stage scale is selected as a scale for the expected values of the thermal environment (ambient temperature, relative humidity, and air flow rate). In Table 4, a five-level scale is selected as an indoor thermal environment and air quality satisfaction evaluation scale. 
Table. 2. Sweat ruler.

\begin{tabular}{|c|c|c|c|c|}
\hline $\begin{array}{c}\text { Degree of } \\
\text { perspiration }\end{array}$ & $\begin{array}{c}\text { Dry skin without } \\
\text { perspiration }\end{array}$ & $\begin{array}{c}\text { No perspiration but } \\
\text { stick }\end{array}$ & $\begin{array}{c}\text { A small amount of } \\
\text { sweat }\end{array}$ & $\begin{array}{c}\text { More } \\
\text { sweat }\end{array}$ \\
\hline Ruler & 0 & 1 & 2 & 3 \\
\hline
\end{tabular}

Table. 3. Expected value scale for thermal environment (ambient temperature, relative humidity, and air flow rate).

\begin{tabular}{|c|c|c|c|}
\hline Expectation indicator & Decrease & constant & Increase \\
\hline Voting value & -1 & 0 & +1 \\
\hline
\end{tabular}

Table. 4. Indoor thermal environment and air quality satisfaction evaluation scale.

\begin{tabular}{|c|c|c|c|c|c|}
\hline Satisfaction & Very dissatisfied & $\begin{array}{c}\text { A little } \\
\text { dissatisfied }\end{array}$ & General & Quite satisfied & Very satisfied \\
\hline Voting value & -2 & -1 & 0 & +1 & +2 \\
\hline
\end{tabular}

\subsection{Test Conditions and Procedures}

According to the monitoring results of indoor thermal environment parameters and experimental conditions on typical summer working days, the setting conditions are as shown in Table 5. The clothing of the subjects were controlled and the subject were asked to sit or move in moderation.

Table. 5. Experimental conditions.

\begin{tabular}{|c|c|c|c|c|}
\hline Lab environment & Temperature & Relative humidity & Wind speed & Clothing thermal resistance \\
\hline 1 & $24^{\circ} \mathrm{C}$ & $50 \%$ & $0.1 \mathrm{~m} / \mathrm{s}$ & $0.35 \mathrm{clo}$ \\
\hline 2 & $26^{\circ} \mathrm{C}$ & $50 \%$ & $0.1 \mathrm{~m} / \mathrm{s}$ & $0.35 \mathrm{clo}$ \\
\hline 3 & $28^{\circ} \mathrm{C}$ & $50 \%$ & $0.1 \mathrm{~m} / \mathrm{s}$ & $0.35 \mathrm{clo}$ \\
\hline 4 & $30^{\circ} \mathrm{C}$ & $50 \%$ & $0.1 \mathrm{~m} / \mathrm{s}$ & $0.35 \mathrm{clo}$ \\
\hline 5 & $32^{\circ} \mathrm{C}$ & $50 \%$ & $0.1 \mathrm{~m} / \mathrm{s}$ & $0.35 \mathrm{clo}$ \\
\hline
\end{tabular}

Twenty students aged 22 to 30 years old were selected, and half of them were men and women. Before the experiment, the subjects were familiarized with the experimental environment and experimental equipment 20 minutes ahead of time, and the filling method of questionnaire was introduced to the subjects. After the subjects having been adapted to the experimental environment, the comfortable physiological experiment was performed, then the thermal environment parameters were recorded and the physiological parameters of the human body were collected.

\section{Establishment of Human Thermal Comfort Model}

\subsection{Experimental Data}

The experimental data obtained from the experiment were analyzed and summarized, which involve in the regularity of skin temperature, skin conductance and heart rate on environmental temperature changes. The effects of room temperature on skin temperature, heart rate and skin conductance were investigated.

\subsection{Establishment of Thermal Comfort Model Based on Partial Least Squares Multiplication Regression Method}

According to the partial least squares regression method, the experimental data and models were analyzed through MATLAB software. Firstly, the mean and standard deviation are obtained, then the correlation coefficient matrix is obtained, and the matrix is normalized. Finally, the results were obtained by partial least squares regression model, as shown in Fig 2.

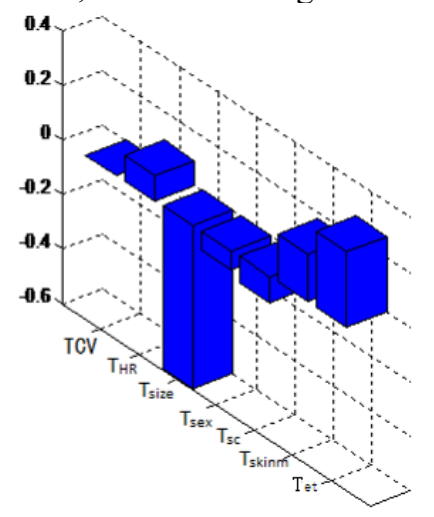

Figure. 2. Model regression coefficient graph based on partial least squares regression.

In Figure 2, $T C V, T_{H R}, T_{\text {size }}, T_{\text {sex }}, T_{s c}, T_{\text {skinm }}$, $T_{e t}$ represent the human body thermal comfort value, heart rate, body type, and gender, skin conductance, skin temperature and ambient temperature respectively.

The least squares regression model between the human thermal comfort value and heart rate, body type, 
gender, skin conductance, skin temperature and ambient temperature can be obtained.

$$
\begin{gathered}
T C V=0.14 T_{H R}-0.60 T_{\text {size }}-0.08 T_{\text {sex }}-0.12 T_{s c} \\
+0.18 T_{\text {skinm }}+0.27 T_{\text {et }}
\end{gathered}
$$

1) The ambient temperature range is $10^{\circ} \mathrm{C} \sim 35^{\circ} \mathrm{C}$;

2) The skin temperature range is $29^{\circ} \mathrm{C} \sim 37.6^{\circ} \mathrm{C}$;

3 ) The gender value is 0 (for women) or 1 (for men);

4) The body type values are $-1,0,1,2,3$, and 4 respectively, which represent the population is lean, normal, overweight, obese, obese and severely obese;

5) The heart rate ranges are from 55 beats/min to 100 beats/min;

6) The skin conductance values range is $10 \mu \Omega \sim 130 \mu \Omega$.

\subsection{Model Establishment Based on ELM-RBF Neural Network}

According to the principle of ELM-RBF neural network, the selected original data about ambient temperature, skin temperature, heart rate, body type, gender and skin conductance are 1450 groups. The specific design steps are as follows:

(1) After the original data is simply summarized, then the data is scrambled and normalized;

(2) 1100 groups of data as training samples were selected randomly, and the remaining 350 groups of data were selected as test samples. The ELM-RBF neural network is a single-layer hidden layer. Through continuous testing, it is found that when the number of neurons in the hidden layer is set to 100 , the model has the best fitting effect;

(3) The thermal comfort model was established by ELM-RBF neural network, and the model was trained 20 times respectively. Finally, it was inverse normalized to obtain the average relative error. The formula of the average relative error is as follows:

$$
\hat{e}=\frac{1}{n} \sum_{i=1}^{n} e_{i}=\frac{1}{n} \sum_{i=1}^{n} \sqrt{\frac{1}{m}\left\|T_{i}-Y_{i}\right\|}
$$

where,

$e_{i}$ - the average error from the ith training;

$T_{i}$ - the expected output vector;

$Y_{i}$ - the actual output vector;

$n$ - the total number of model establishment.

According to the above steps, the ambient temperature, skin temperature, heart rate, body type, gender and skin conductance were considered as input and the thermal comfort value of the human body was considered as output. The test results and the average relative error of the model are as shown in Figures 3 and 4 respectively.

For Figure 3, the solid blue line indicates the true value of human thermal comfort, and the red dotted line indicates the predicted value. Clearly, most of the predicted values are in agreement with the true values, which are calculated by the average error formula. As shown in Figure 4, it can be seen that the average error is about 0.062 , and the goodness of fit is 0.8909 , which indicate that the predicting effect is better.

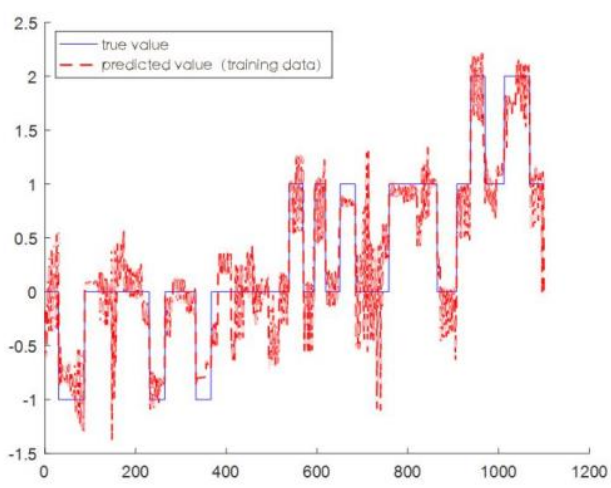

Figure. 3. Test results based on ELM-RBF neural network.

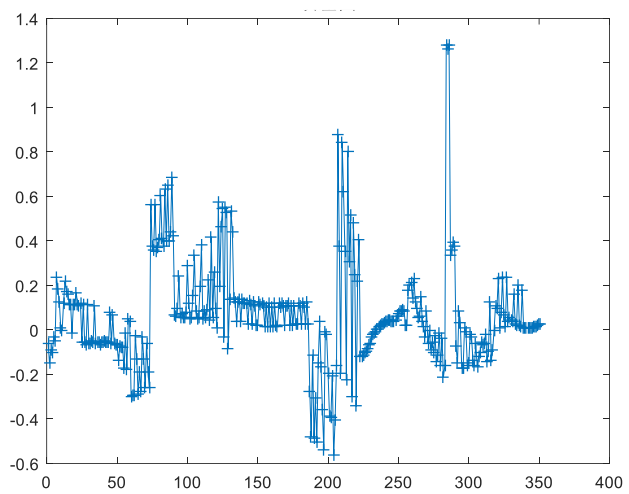

Figure. 4. Average error graph based on ELM-RBF.

\section{Results and Discussion}

In order to compare the thermal comfort model based on partial least squares regression and the thermal comfort model based on ELM-RBF neural network with the classical PMV model, the model prediction map was drawn. Figures 5-7 are the thermal comfort model of the ELM-RBF neural network, the thermal comfort model based on partial least squares regression and the prediction maps of the classical PMV model respectively.

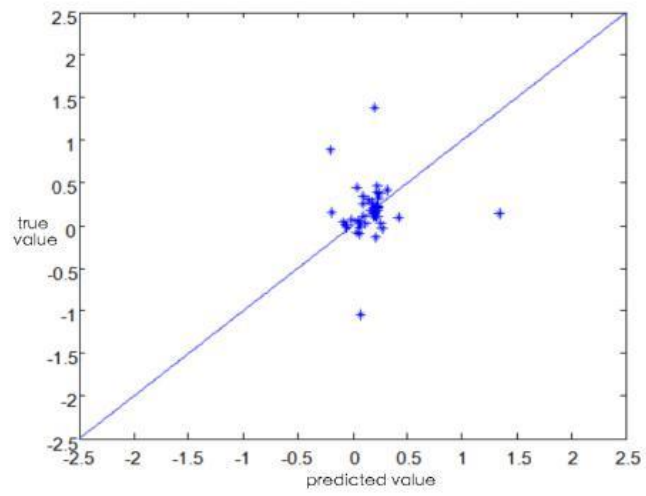

Figure. 5. Thermal comfort model based on ELM-RBF neural network. 


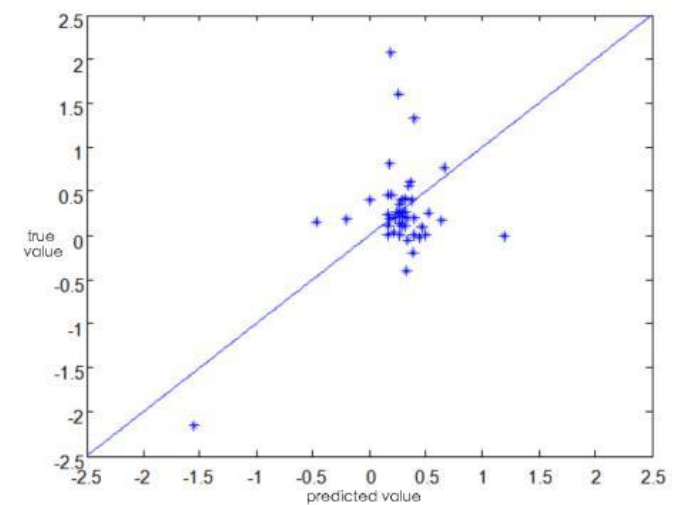

Figure. 6. Thermal comfort model based on partial least squares regression.

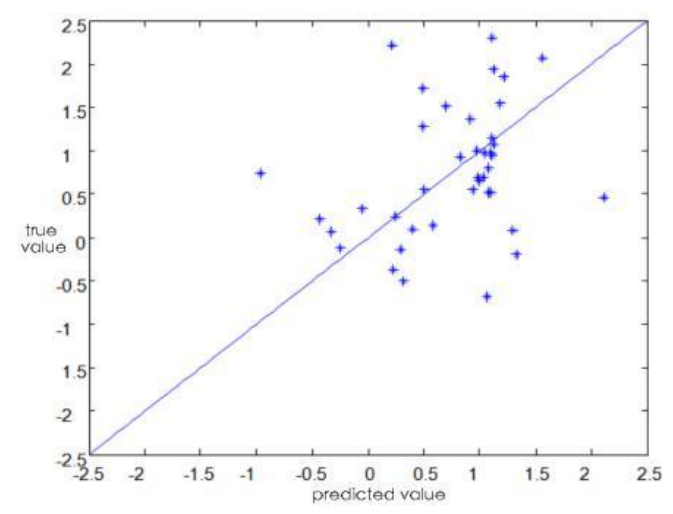

Figure. 7. Classic PMV model recognition prediction map.

For the thermal comfort model established based on the ELM-RBF neural network, it can be seen that a few points are far from the diagonal, the other points are near the diagonal, which indicate the difference between the combined value and the true value is small. For the thermal comfort model established based on partial least squares regression, most of the points are near the diagonal, but the number is less than the points of the thermal comfort model established based on ELM-RBF neural network. In the classic PMV model, the points are scattered around the diagonal, and there are very few points that fall near the diagonal. Therefore, it can be concluded that the fitting effect of the thermal comfort model based on the ELM-RBF neural network is better. The fitting results of the thermal comfort model based on partial least squares regression is not satisfactory. And the fitting effect based on classic PMV model is not satisfactory too. Compared with the PMV classic model, the two individual physiological evaluation models established based on individual differences are feasible, and the thermal comfort model based on the ELM-RBF neural network is better.

\section{Conclusion}

The thermal comfort evaluation models based on individual differences were established by partial least squares regression and ELM-RBF neural network. The evaluating results of the two established models were compared with the classical PMV model and the pros and cons of the model were analyzed. The evaluation model established by ELM-RBF neural network can overcome the drawbacks of subjective evaluation and provide theoretical guidance for objective evaluation of human thermal comfort. In the end, it achieves the purpose of building energy saving.

\section{Acknowledgements}

This research was funded by National Key Research and Development Program of China No. 2017YFC0704100 (New Generation Intelligent Building Platform Techniques).

\section{References}

1. Yulong Fang. Effects of Indoor Thermal Environment on Human Thermal Comfort. [J] House, (11): 175(2019)

2. Yinghui Ding, Bin Chen, Xing Chen. Summary of the development of thermal comfort model research. [J] HVAC, (09): 29-34(2006)

3. Yu Huang, Jianlei Niu. A review of the advance of HVAC technologies as witnessed in ENB publications in the period from 1987 to 2014. Energy and Buildings, 130(15): 33-45(2016)

4. Yingdong He, Nianping Li, Jinqing Peng, Wenjie Zhang, Yiru Li. Field study on adaptive comfort in air conditioned dormitories of university with hothumid climate in summer. Energy and Buildings, 119: 1-12(2016)

5. Jiayi Li, Jingxian $\mathrm{Xu}$, Jun Li. Advances in the study of thermal comfort model modification based on physiological differences in human body[J]. Shanghai Textile Technology, 47(04): 12-18(2019)

6. Zhou Xin. Multi-node thermal comfort model based on Chinese thermal characteristics[D]. Shanghai jiaotong university (2015)

7. Alfano, FRD, Palella, BI, Riccio, G. Thermal Environment Assessment Reliability Using Temperature Humidity Indices. Industrial Health, 49(1): 95-106(2011)

8. Sun, Yu Hang, Jia Yu, Wang Yu. A Review of Experimental Study on Physiological Parameters in the Field of Thermal Comfort[J]. Building thermal ventilation and air conditioning, 37(12): 6064+21(2018)

9. Hao Wu. Research on indoor thermal comfort control system based on Smith prediction and feedforward decoupling[D]. Anhui JianZhu University (2015)

10. Ali Ghahramani, Guillermo Castro, Burcin BecerikGerber, et al. Infrared thermography of human face for monitoring thermoregulation performance and estimating personal thermal comfort[J]. Building and Environment, 109: 1 11(2016) 